\title{
Highly Stable Periodic Structures Using Nonlinear Laser Lithography
}

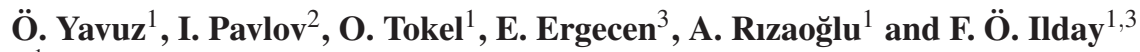 \\ ${ }^{1}$ Department of Electrical and Electronics Engineering, Bilkent University, 06800 Ankara, Turkey \\ ${ }^{2}$ Department of Physics, Bilkent University, 06800 Ankara, Turkey \\ ${ }^{3}$ Department of Electrical and Electronics Engineering, Middle East Technical University, 06800 Ankara, Turkey
}

\begin{abstract}
Nonlinear laser lithography (NLL) emerged as a novel surface structuring method allowing longrange periodic order. We present mathematical formalism for NLL, analysis of structure stability to perturbations and a way to control final tiling patterns.
\end{abstract}

\section{Introduction}

Just five years after invention of the laser, laser induced periodic structures (LIPSS) had been reported [1]. However, the structure period is not very uniform in LIPSS. Recently, with nonlinear laser lithography (NLL), long range ordered periodic surface structures had been maintained [2]. Here, we present a rigours mathematical model for NLL, effect of the defects on the surface structure uniformity, and finally, a way to gain control over the final surface structures with changing initial conditions of the surface.

\section{Mathematical Model of NLL}

In NLL, long-range ordered periodic surface structures are formed interplay between three mechanisms which are positive feedback, nonlinearity, and negative feedback. Each infinitesimal point on the surface of target material scatter the light proportional to its height. Each scatterer, scatters the light as a dipole radiation and creates positive feedback on the overall system. The points where, interference of electric field caused by all scatterers becomes maximum, start to ablate and react with the surrounding molecules. Ignoring the near field terms ( $1 / r^{2}$ and $1 / r^{3}$ terms in the dipole radiation), scattered electric fields can be formalised as:

$$
\begin{gathered}
E_{x, \text { scat }}(x, y)=\gamma \int_{S} h\left(x^{\prime}, y^{\prime}\right)\left(\cos ^{2} \theta E_{x}\left(x^{\prime}, y^{\prime}\right)-\right. \\
\left.\cos \theta \sin \theta E_{y}\left(x^{\prime}, y^{\prime}\right)\right) \frac{e^{j k r}}{r} d x^{\prime} d y^{\prime} \\
E_{y, \text { scat }}(x, y)=\gamma \int_{S} h\left(x^{\prime}, y^{\prime}\right)\left(-\cos \theta \sin \theta E_{x}\left(x^{\prime}, y^{\prime}\right)+\right. \\
\left.\sin ^{2} \theta E_{y}\left(x^{\prime}, y^{\prime}\right)\right) \frac{e^{j k r}}{r} d x^{\prime} d y^{\prime}
\end{gathered}
$$

,where $E_{x}\left(x^{\prime}, y^{\prime}\right)$ and $E_{y}\left(x^{\prime}, y^{\prime}\right)$ are the incident electric field components, $h\left(x^{\prime}, y^{\prime}\right)$ is the surface height profile, $\cos \theta=\frac{y-y^{\prime}}{r}, \sin \theta=\frac{x-x^{\prime}}{r}, r^{2}=\left(x-x^{\prime}\right)^{2}+\left(y-y^{\prime}\right)^{2}$ and $\gamma$ is constant. Nonlinearity of NLL from strongly nonlinear response of NLL to positive feedback, i.e. relative height growth per pulse is nonlinear function of number of pulses. Finally, negative feedback emerges due to the extinction of one of the species in the environment (either target material or surrounding molecules).

\section{Simulation and Experimental Results}

In experiments we have used an ultrafast Yb-fibre laser which has a 170 fs pulse duration, $1 \mathrm{MHz}$ repetition rate. The target material in our experiments is $\mathrm{Ti}$ thin film which has $50 \mathrm{~nm}$ thickness on the glass substrate. We are creating $\mathrm{TiO}_{2}$ on the top of the Ti sample which means our reactant is $\mathrm{O}_{2}$ molecules in the ambient air. Simulation and experimental results for vertical polarisation can be seen in Fig. 1 (a) and (b) respectively. Similarly, simulation and experimental results for horizontal polarisation can be seen in Fig. 1 (c) and (d) respectively.

(a)
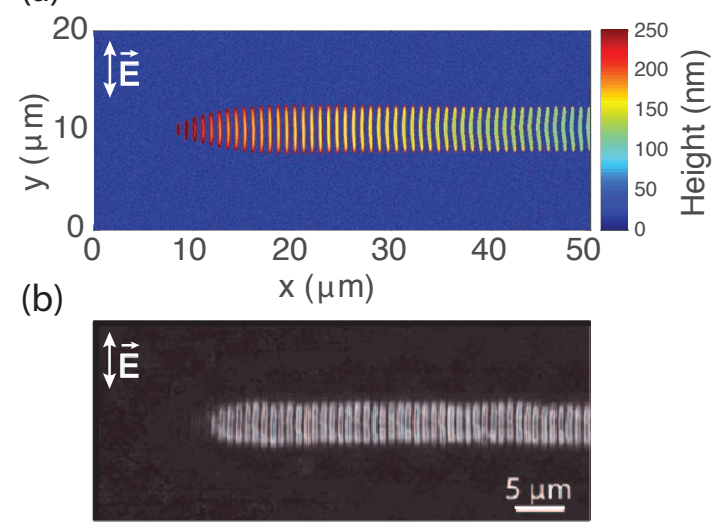

(c)
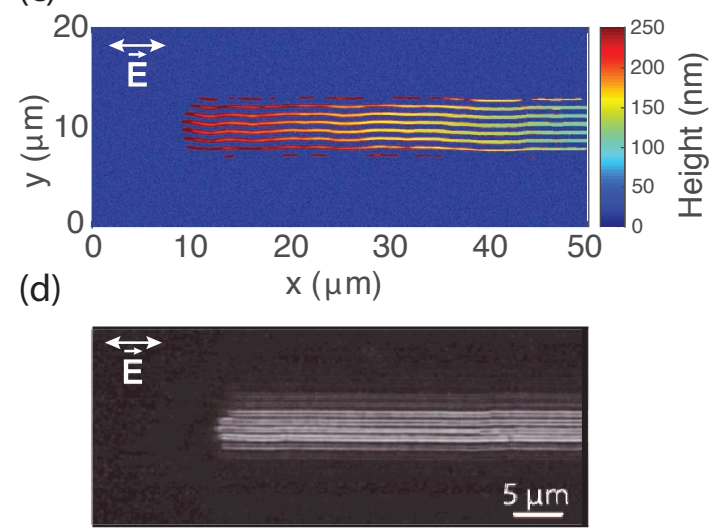

Fig. 1 (a) Simulation results of scanning along the $x$-direction with vertically polarised laser beam. (b) Experimental realisation of simulations shown in (a). (c) Simulation results of scanning along the $x$-direction with horizontally polarised laser beam. (d) Experimental realisation of simulations shown in (c).

According to the simulation and experimental results shown in Fig. 1, the lines always emerges parallel to the polarisation. 


\section{Effect of Perturbations on the Periodicity}

In order to analyse the stability of our surface structures done with linear polarisation we introduced additional defects with various sizes to the scanning path. The response of surface structures to these defects can be seen in Fig. 2. Effect of defects on the vertical polarisation can be seen in Fig. 2. (a) and (b). Similarly, effect of defects on the horizontal polarisation can be seen in Fig. 2 (c) and (d). Although, we did not include the intermediate diameter values in Fig. 2, the stability of the structures decreases gradually. For both polarisation types, large defects disturb the uniformity of the structures (Fig. 2 (b) and (d)) because of the feedback mechanisms explained in mathematical model. However, we observed that scanning along the polarisation direction, makes the structures more stable against perturbations/defects on the surface.

(a)
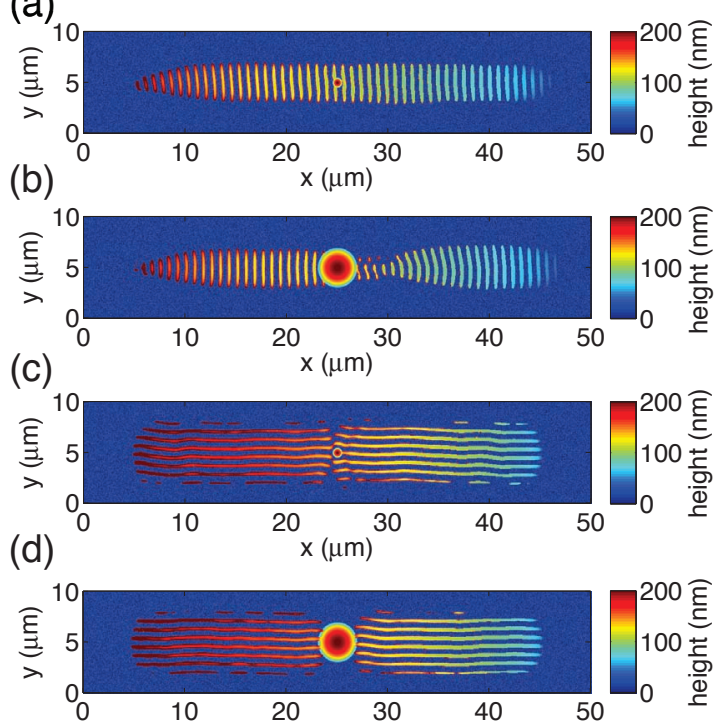

Fig. 2 (a), (b) Simulation results of scanning along the $x$-direction with vertically polarised laser beam. (a) Defect diameter $1 \mu \mathrm{m}$ and (b) effect diameter $5 \mu \mathrm{m}$. (c), (d) Simulation results of scanning along the $x$-direction with horizontally polarised laser beam. (c) Defect diameter $1 \mu \mathrm{m}$ and (d) effect diameter $5 \mu \mathrm{m}$.

\section{Controlling Laser-induced Self-organised Patterns via Engineered Defects}

Having observed the surface structures with linear polarisation on rough (empty) surface, we introduced prestructured surfaces instead of rough surface. First, we created surfaces with horizontal lines using NLL, and then we scanned the surface with perpendicularly polarised ( $y$-polarised) light on $x$-direction. This kind of scanning creates the checkerboard resembling pattern as in Fig. 3 (a). Fig. 3 (b) is the experimental realisation of Fig. 3 (a). In addition to the horizontal lines, we added an additional line defect which is perpendicular to the pre-conditioned surface $(x=10 \mu \mathrm{m})$. Starting from this line defect, scanning in the $x$-direction yields mesh pattern as in Fig. 3 (c). The experimental result corresponding Fig. 3 (c) is Fig. 3 (d).
The emergence of the patterns in NLL is intrinsically a stochastic process. However there is a causal relationship between the final pattern and the initial surface roughness. The exploitation of this effect is possible by manually introducing defects to influence the final pattern in a predictable manner.

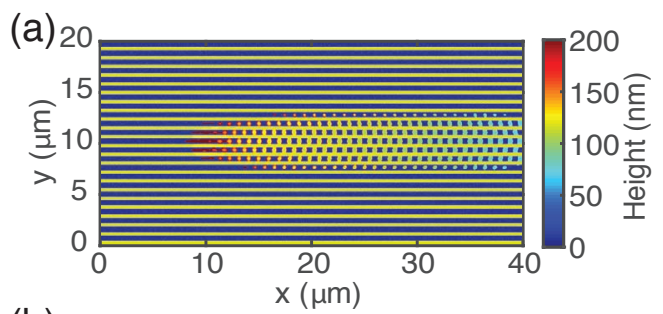

(b)
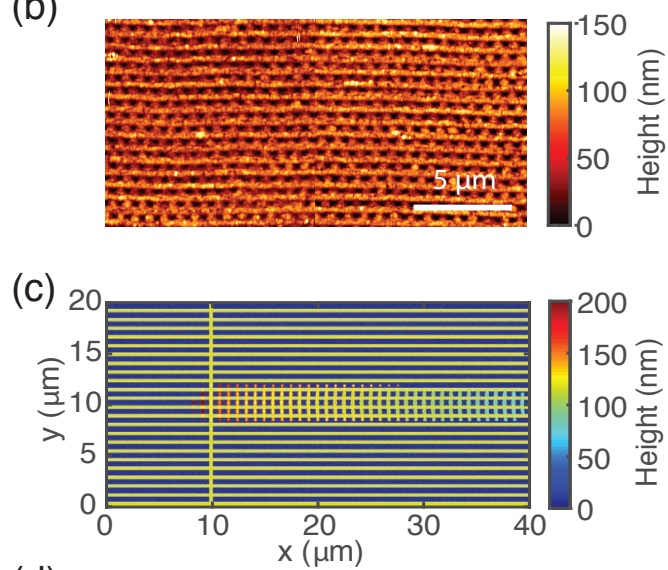

(d)

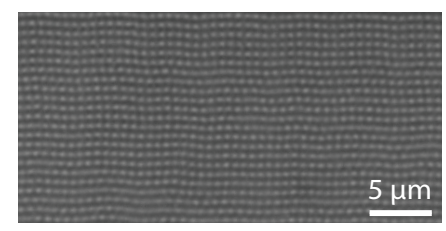

Fig. 3 (a) Surface pattern obtained by scanning the laser from $(x, y)=$ $(10,10) \mu \mathrm{m}$ to $(40,10) \mu \mathrm{m}$. (b) Surface pattern obtained by scanning the laser from $(\mathrm{x}, \mathrm{y})=(10,10) \mu \mathrm{m}$ to $(40,10) \mu \mathrm{m}$. (c) AFM image of the experiments corresponding to (a). (d) Optical microscopy image of the experiment corresponding to (b). The laser has a beam diameter of $10 \mu \mathrm{m}$

\section{Conclusion}

In conclusion, here, we present a mathematical model for NLL and the simulation results agree with the experiments. Furthermore, we have analysed stability of the surface structures to perturbations (defects) via simulations. Finally, we have shown a novel method to change final surface pattern via engineering initial surface conditions. Notably, more complex periodic surface tilings, such as honeycomb lattices or quasicrystals might be obtained, and controlled by engineering the initial surface conditions.

\section{References}

[1] M. Birnbaum, "Semiconductor surface damage produced by ruby lasers," J. Appl. Phys. 36, 3688 (1965).

[2] B. Öktem, I. Pavlov, S. llday, H. Kalaycıŏ̆lu, A. Rybak, S. Yavaş, M. Erdoğan, and F. Ö. 1lday, "Nonlinear laser lithography for indefinitely large-area nanostructuring with femtosecond pulses," Nature Photon. 7, 897 (2013). 